\title{
RINGS WITH PROJECTIVE SOCLE
}

\author{
W. K. NICHOLSON AND J. F. WATTERS
}

(Communicated by Donald Passman)

\begin{abstract}
The class of rings with projective left socle is shown to be closed under the formation of polynomial and power series extensions, direct products, and matrix rings. It is proved that a ring $R$ has a projective left socle if and only if the right annihilator of every maximal left ideal is of the form $f R$, where $f$ is an idempotent in $R$. This result is used to establish the closure properties above except for matrix rings. To prove this we characterise the rings of the title by the property of having a faithful module with projective socle, and show that if $R$ has such a module, then so does $M_{n}(R)$. In fact we obtain more than Morita invariance. Also an example is given to show that $e R e$, for an idempotent $e$ in a ring $R$ with projective socle, need not have projective socle. The same example shows that the notion is not left-right symmetric.
\end{abstract}

1. Introduction. We recall that a study of rings with projective socle and containing no infinite sets of orthogonal idempotents was make by Gordon [8]. In the same paper he showed that $S=\operatorname{Soc}_{R} R$ is projective and essential if and only if $S$ has zero right annihilator. More recently Baccella [4] has provided a number of necessary and sufficient conditions for $R$ to have projective socle, conditions originally given by Manocha [9] when the socle was known to be essential. One such condition is that $\operatorname{soc}_{R} R$ is nonsingular. However all these statements explicitly involve the socle. Our characterizations are somewhat different in that they involve either the maximal left ideals of $R$ or modules with projective socles. Theorem 2.4 provides a number of conditions equivalent to the statement that $R$ has a projective socle.

As examples of rings with projective socles we have semiprime rings, nonsingular rings, and PP-rings. It is well known that $R$ is a semiprime ring if and only if $R[x]$ is semiprime. It is an easy consequence of a theorem of Shock [11, Theorem 2.7] that the same holds for nonsingular rings. For PP-rings Armendariz [2] has shown that given a reduced ring $R, R$ is a PP-ring if and only if $R[x]$ is a PP-ring. The same result holds for Baer rings and Burgess [6] remarks in his review of [2] that the Baer ring result is true for $R[[x]]$ whilst for PP-rings it is false. Further, the polynomial results are not true if the restriction to reduced rings is removed. In Theorem 3.1 we show that if $R$ has a projective socle, then so does $R[x]$ (and $R[[x]]$ ), but the converse is false.

In the final section we establish Morita invariance. Our proof of this uses an extension of an idea of Amitsur [1]. We call a module a PS-module if it has

Received by the editors August 4, 1986

1980 Mathematics Subject Classification (1985 Revision). Primary 16A50; Secondary 16A89, $16 \mathrm{~A} 05$.

Key words and phrases. Projective socle, polynomial rings, Morita invariance.

Research supported by NSERC grant A8075. 
projective socle. A ring $R$ has projective socle if and only if it has a faithful PSmodule; this is part of Theorem 2.4. If $\left[\begin{array}{cc}R & V \\ W & S\end{array}\right]$ is a Morita context and $M$ is a left $R$-module, there is a related $S$-module $M^{\circ}$ which inherits some properties from $M$ under mild restrictions on the context and we show in Proposition 4.4 that being a PS-module is such a property. Morita invariance is an easy consequence of this.

Throughout the paper, all rings have a unity and all modules are unitary. Modules are left modules unless otherwise stated and module homomorphisms are written opposite the scalars. The left and right annihilators of a subset $X$ of a ring are written $\ell(X)$ and $r(X)$ respectively.

\section{Characterizations.}

2.1 Definition. A left $R$-module ${ }_{R} M$ is called a PS-module if every simple submodule is projective; equivalently if $\operatorname{soc}\left({ }_{R} M\right)$ is projective.

2.2 EXAMPLES. 1. If $\operatorname{soc}\left({ }_{R} M\right)=0$ then $M$ is a PS-module.

2. Any projective semisimple module is a PS-module.

3. Every regular module (in the sense of Zelmanowitz [12]) is a PS-module because every principal submodule is a projective summand.

4. Every nonsingular module is a PS-module because nonsingular simple modules $K$ are projective. Indeed, if $0 \neq k \in K$ then $\ell(k)$ is not essential in $R$ so $\ell(k) \cap X=0$ for some left ideal $X \neq 0$. It follows that $R=\ell(k) \oplus X$ so $K \cong X$ is projective.

5 . The class of PS-modules is closed under direct sums, submodules, and essential extensions.

2.3 Definition. $A$ ring $R$ is called a (left) $P S$-ring if ${ }_{R} R$ is a PS-module.

The PS-rings are defined by the requirement that their simple left ideals are projective. However the next result gives several characterizations involving maximal left ideals.

2.4 THEOREM. The following are equivalent for a ring $R$ :

(1) $R$ is a PS-ring.

(2) $R$ has a faithful PS-module.

(3) If $L$ is a maximal left ideal of $R$ then either $r(L)=0$ or $L=R e$ where $e^{2}=e \in R$.

(4) If $L$ is an essential maximal left ideal of $R$ then $r(L)=0$.

(5) If $L$ is a maximal left ideal of $R$ then $r(L)=f R$ where $f^{2}=f \in R$.

(6) If $L$ is a maximal left ideal of $R$ and $t \in r(L)$, then $t \in r(L) t$ (that is $R / r(L)$ is flat as a right $R$-module [3]).

(7) Every simple module ${ }_{R} K$ is either projective or $K^{d}=0$ where the dual module is denoted by $K^{d}=\operatorname{hom}(K, R)$.

(8) $\operatorname{soc}\left({ }_{R} R\right)$ is projective.

PROOF. (1) $\Rightarrow(2)$. This is clear.

(2) $\Rightarrow(3)$. Let ${ }_{R} M$ be a faithful PS-module and let $L \subseteq R$ be a maximal left ideal. If $r(L) \neq 0$ write $T=r(L)$ so that $L T=0$. On the other hand $R T \neq 0$ so $R T M \neq 0$ by hypothesis, say $R m \neq 0$ with $m \in T M$. Thus $L \subseteq \ell(m) \neq R$ so $L=\ell(m)$. But then $R / L \cong R m$ is projective by hypothesis and (3) follows.

$(3) \Rightarrow(4)$. This is clear.

$(4) \Rightarrow(5)$. This is because maximal left ideals are either essential or summands. 
(5) $\Rightarrow(6)$. If $t \in \mathscr{R}(L)$ as in (6) let $\mathscr{R}(L)=f R, f^{2}=f$. Then $t=f t \in \mathscr{R}(L) t$.

(6) $\Rightarrow(3)$. If $r(L)=0$ take $f=0$. Otherwise let $0 \neq a \in r(L)$. Then $a \in r(L) a$, say $a=f a$ with $f \in r(L)$. We claim that $1-f \in L$. For if not then, since $L$ is maximal, $1=t+r(1-f), t \in L$. Then $a=t a+r(a-f a)=0$, a contradiction. So $R(1-f) \subseteq L$ whence $(1-f) f \in L f=0$, that is $f^{2}=f$. On the other hand $L f=0$ gives $L=L(1-f) \subseteq R(1-f)$ so $L=R(1-f)$. Thus $r(L)=f R$.

(3) $\Rightarrow(7)$. Let $K=R k$ be simple so that $L=\ell(k)$ is maximal and $R / L \cong K$. If $L=R e, e^{2}=e$, then $K \cong R(1-e)$ is projective. If $\mu(L)=0$ let $\lambda \in K^{d}$. If $k \lambda=a \in R$ then $L a=L(k \lambda)=(L k) \lambda=0 \lambda=0$, so $a=0$. This means $\lambda=0$ and so proves $K^{d}=0$.

(7) $\Rightarrow(8)$. This is clear since $K^{d} \neq 0$ for all left ideals $K \neq 0$.

$(8) \Rightarrow(1)$. This is clear.

2.5 EXAMPLES. 1. Every semiprime ring is a PS-ring. In fact it is enough that $R$ has no nilpotent simple left ideals. On the other hand, if $\Delta$ is a division ring and $R=\left[\begin{array}{cc}\Delta & \Delta \\ 0 & \Delta\end{array}\right]$ then $\operatorname{soc}\left({ }_{R} R\right)=\left[\begin{array}{cc}\Delta & \Delta \\ 0 & 0\end{array}\right]$ is projective (and homogeneous) but $J(R)=\left[\begin{array}{ll}0 & \Delta \\ 0 & 0\end{array}\right]$ is nilpotent.

2. Every PP-ring is a PS-ring (where a PP-ring has every principal left ideal projective). In particular every Baer ring is a PS-ring (where $R$ is Baer if every left (or right) annihilator is generated by an idempotent). Of course this follows directly from (5) of Theorem 2.4 .

3. Every left nonsingular ring is a PS-ring by (4) of Theorem 2.4 since these rings are characterized by $r(L)=0$ for every essential left ideal $L$. In fact $R$ is a PS-ring if $Z\left(\operatorname{soc}_{R} R\right)=0$ by 4 of Example 2.2.

4. A ring for which every simple singular module is injective is a PS-ring (these are the GV-rings of Baccella [5]). In fact if $K$ is a simple left ideal it is either projective or $Z(K)=K$, in which case it is a summand of $R$ (being injective) and so again is projective. Of course, this shows that $V$-rings are PS-rings.

5. If $r[J(R)]=0$ then $R$ is a PS-ring. In fact $J(R) \subseteq L$ for every maximal left ideal so $r(L)=0$.

The next result will be used to give an example of a (left) PS-ring which is not a right PS-ring.

2.6 LEMMA. Let $R$ and $S$ be rings, let ${ }_{R} V_{S}$ be a bimodule, and consider the $\operatorname{ring} C=\left[\begin{array}{cc}R & V \\ 0 & S\end{array}\right]$.

(1) If $C$ is a PS-ring then $R$ is a PS-ring.

(2) $M$ is a maximal left ideal of $C$ if and only if either $M=\left[\begin{array}{ll}L & V \\ 0 & S\end{array}\right]$, where $L$ is a maximal left ideal of $R$, or $M=\left[\begin{array}{cc}R & V \\ 0 & K\end{array}\right]$ where $K$ is a maximal left ideal of $S$.

ProOF. (1) If $L \subseteq R$ is maximal then $\left[\begin{array}{ll}L & V \\ 0 & S\end{array}\right]$ is maximal in $C$. If $r_{C}\left[\begin{array}{ll}L & V \\ 0 & S\end{array}\right]=0$ then $r_{R}(L)=0$; while if $\left[\begin{array}{ll}L & V \\ 0 & S\end{array}\right]=C g, g^{2}=g$, and $g=\left[\begin{array}{ll}e & v \\ 0 & f\end{array}\right]$, then $e^{2}=e \in R$ and $R e=L$.

(2) Given $M$ as in (2) let $K_{0}=\left\{s \in S \mid\left[\begin{array}{ll}r & v \\ 0 & s\end{array}\right] \in M\right.$ for some $\left.r, v\right\}$. This is a left ideal of $S$ so either $K_{0}=S$ or $K_{0} \subseteq K \subset S, K$ maximal. In the latter case $M=$ $\left[\begin{array}{ll}R & V \\ 0 & K\end{array}\right]$; in the former case $\left[\begin{array}{ll}0 & V \\ 0 & S\end{array}\right]=C\left[\begin{array}{ll}0 & 0 \\ 0 & 1\end{array}\right] \subseteq M$ so let $L_{0}=\left\{r \in R \mid\left[\begin{array}{ll}r & 0 \\ 0 & 0\end{array}\right] \in M\right\}$. Then $L_{0}$ is a left ideal of $R$ and $L_{0} \neq R$ so, if $L_{0} \subseteq L \subset R$ with $L$ maximal, it follows as before that $M=\left[\begin{array}{ll}L & V \\ 0 & S\end{array}\right]$. 
2.7 ExAMPLE. Let $S=\mathbf{Z}_{2}[x] /\left(x^{2}\right)$ and let $R=\left[\begin{array}{cc}\mathbf{z}_{2} & S \\ 0 & S\end{array}\right]$. Then

(1) $R$ is a (left) PS-ring which is not a right PS-ring.

(2) If $e=\left[\begin{array}{ll}0 & 0 \\ 0 & 1\end{array}\right]$ then $e^{2}=e \in R$ and $e R e$ is not a left PS-ring.

PROOF. (1) We have $S=\mathbf{Z}_{2} \oplus \mathbf{Z}_{2} t, t^{2}=0$. The only maximal left ideals of $\mathbf{Z}_{2}$ and $S$ are 0 and $S t$ respectively so $\left[\begin{array}{ll}0 & S \\ 0 & S\end{array}\right]=R\left[\begin{array}{ll}0 & 0 \\ 0 & 1\end{array}\right]$ and $\left[\begin{array}{cc}\mathbf{Z}_{2} & S \\ 0 & S t\end{array}\right]$ are the only maximal left ideals of $R$, and $r\left[\begin{array}{cc}\mathbf{z}_{2} & S \\ 0 & S t\end{array}\right]=0$. On the other hand, $X=\left[\begin{array}{cc}\mathbf{z}_{2} & S \\ 0 & t S\end{array}\right]$ is a maximal right ideal of $R$ and $\ell(X)=\left[\begin{array}{ll}0 & t S \\ 0 & t S\end{array}\right]$ is not of the form $R f, f^{2}=f$.

(2) We have $e R e \cong S$, a commutative local ring with unique maximal ideal $J=t S$. Clearly $r(J)=t S$ is not of the form $f S, f^{2}=f$.

3. Closure properties. If $R$ is a PP-ring it is known that $R[x]$ need not be a PP-ring (although this is true if $R$ is reduced-see [2]). However we do have the following result for PS-rings. We use the notation $R[[x]]$ to denote the ring of formal power series.

\subsection{THEOREM. If $R$ is a $P S$-ring so also are $R[x]$ and $R[[x]]$.}

PROOF. We prove the result for $R[x]$ only; the proof for $R[[x]]$ is similar. If $L$ is a maximal ideal of $R[x]$ we show that $r(L)=e R[x]$ for an idempotent $e^{2}=e \in R[x]$. Let $I$ denote the left ideal of $R$ consisting of the set of all trailing coefficients of polynomials in $L$ with 0 adjoined. If $I=R$ then $r(L)=0$. So we assume $I \neq R$ and show that $I$ is a maximal left ideal of $R$.

Let $a \in R-I$. Then $a \notin L$ so $R[x]=L+R[x] a$. Hence, considering constant terms, $1=l_{0}+r_{0} a$ in $R$ where $l_{0}+l_{1} x+l_{2} x^{2}+\cdots \in L$. If $l_{0}=0$ then $1 \in R a$ and so $I+R a=R$. If $l_{0} \neq 0$ then $l_{0} \in I$ and again $I+R a=R$. Hence $I$ is a maximal left ideal of $R$.

By hypothesis $r(I)=e R$ with $e^{2}=e \in R$, and we complete the proof by showing that $r(L)=e R[x]$. If $L e \nsubseteq L$ then $R[x]=L+L e$ so (by considering constant terms) $1 \in I$, a contradiction. Hence $L e \subseteq L$ and it follows (by considering trailing coefficients of elements of $L e)$ that $L e=\overline{0}$. Hence $r(L) \supseteq e R[x]$.

To establish the opposite inclusion take $g \in r(L)-e R[x]$, say $g=b_{n} x^{n}+$ $b_{n+1} x^{n+1}+\cdots$ where $b_{n} \neq 0$. If $b_{n} \in e R$ then $g-b_{n} x^{n} \in \mu(L)$ so we may assume $b_{n} \notin e R$. Then if $h \in L$ we have $h g=0$ so (considering trailing terms) we find that $I b_{n}=0$. This means $b_{n} \in r(I)=e R$, a contradiction. Thus $r(L)=e R[x]$ as required.

The converse to this result is false.

3.2 EXAMPLE. If $R=\mathbf{Z}_{4}$ then $R[x]$ and $R[[x]]$ are $P S$-rings but $R$ is not a PS-ring.

ProOF. It is not difficult to show that $J(R[x])=2 R[x]$ and $J(R[[x]])=2 R[[x]]+$ $x R[[x]]$. Hence if $L$ is a maximal (left) ideal of $R[x]$ (or $R[[x]]$ ) then $L$ contains an element with 1 as trailing coefficient. Hence $r(L)=0$. Thus $R[x]$ and $R[[x]]$ are PS-rings by (3) of Theorem 2.4.

3.3 THEOREM. Let $R=\prod_{i \in I} R_{i}$ be a direct product of rings. Then $R$ is a $P S$-ring if and only if $R_{i}$ is a PS-ring for all $i \in I$.

ProOF. Let $L$ be a maximal left ideal of $R$ and, for each $t \in I$, define

$$
L_{t}=\left\{r \in R_{t} \mid\left\langle r_{i}\right\rangle \in L \text { where } r_{i}=0 \text { for } i \neq t \text { and } r_{t}=r\right\}
$$


Then $L_{t}$ is a left ideal of $R_{t}$ for each $t$ and $L \subseteq \prod_{i} L_{i}$. If some $L_{t} \neq R_{t}$ suppose $L_{t} \subseteq$ $M_{t} \subset R_{t}, M_{t}$ maximal. Taking $M_{i}=R_{i}$ for $i \neq t$ gives $L \subseteq \prod L_{i} \subset \prod M_{i} \neq R$, contradicting the maximality of $L$. Thus $L_{t}=R_{t}$ or $L_{t}$ is maximal in $R_{t}$ for every $t$. Either way our hypotheses guarantee that $r\left(L_{t}\right)=f_{t} R_{t}$ where $f_{t}^{2}=f_{t} \in R_{t}$ for all $t$. If $f=\left\langle f_{i}\right\rangle$ we claim $r(L)=f R$. It is clear that $L f \subseteq\left(\prod L_{i}\right) f=0$ so $f R \subseteq r(L)$. On the other hand, if $L a=0$ where $a=\left\langle a_{i}\right\rangle \in R$ then $L_{t} a_{t}=0$ for each $t$ so $a_{t} \in f_{t} R_{t}$. It follows that $a \in f R$ so $r(L)=f R$. Hence $R$ is a PS-ring by (5) of Theorem 2.4.

Conversely, if $R$ is a PS-ring and $L_{t}$ is a maximal left ideal of $R_{t}$, take $L_{i}=R_{i}$ for all $i \neq t$. Then $\prod_{i} L_{i}$ is a maximal left ideal of $R$ so $r(L)=f R$ where $f^{2}=f \in R$. If $f=\left\langle f_{i}\right\rangle$ it follows that $r\left(L_{t}\right)=f_{t} R_{t}$, so $R_{t}$ is a PS-ring for each $t \in I$.

We do not know if a subdirect product of PS-rings is again a PS-ring. However, for PS-rings which are also normal (that is, every idempotent is central [7]) we do have subdirect closure. The next result is needed.

3.4 Proposition. Let $R$ be a normal ring. Then $R$ is a PS-ring if and only if $J\left(\operatorname{soc}_{R} R\right)=0$.

ProOF. Let $R$ be a PS-ring. If $J\left(\operatorname{soc}_{R} R\right) \neq 0$ then there is a minimal left ideal $M$ of $R$ with $M \subseteq J(R)$. But $M$ is projective by hypothesis, so $M \cong R e$ for some $0 \neq e^{2}=e \in R$. This means $(1-e) M=0$ since $e$ is central so that $M \subseteq e R=R e$. But then $M=R e$ (because $R e$ is minimal) so $e \in J(R)$, a contradiction. Therefore $J\left(\operatorname{soc}_{R} R\right)=0$.

Conversely, if $M$ is a minimal left ideal of $R$ then $J\left(\operatorname{soc}_{R} R\right)=0$ implies $M^{2} \neq 0$ so $M=R e$ where $e^{2}=e \in R$. Thus $M$ is projective and $R$ is a PS-ring.

3.5 PROPOSITION. A subdirect product of normal PS-rings is again a normal PS-ring.

ProOF. Let $R / A_{i}$ be a normal PS-ring for each $i \in I$ where $\bigcap_{i \in I} A_{i}=0$. It is clear that $R$ is normal. If $M$ is a minimal left ideal of $R$ then $M \nsubseteq A_{i}$ for some $i$ so $\left(M+A_{i}\right) / A_{i}$ is a minimal left ideal of $R / A_{i}$. It follows from Proposition 3.4 that $M^{2} \nsubseteq A_{i}$ and so $M^{2}=M$. Therefore $J\left(\operatorname{soc}_{R} R\right)=0$ and $R$ is a PS-ring, again by Proposition 3.4.

4. Morita invariance. A Morita context is a four-tuple $\left[\begin{array}{cc}R & V \\ W & S\end{array}\right]$ where $R$ and $S$ are rings and ${ }_{R} V_{S}$ and ${ }_{S} W_{R}$ are bimodules, together with multiplications $V \times W \rightarrow$ $R$ and $W \times V \rightarrow S$ which induce bimodule homomorphisms $V \otimes_{S} W \rightarrow R$ and $W \otimes_{R} V \rightarrow S$, and which satisfy the conditions $(v w) v_{1}=v\left(w v_{1}\right)$ and $(w v) w_{1}=$ $w\left(v w_{1}\right)$ for all $v, v_{1}$ in $V$ and all $w, w_{1}$ in $W . R$ and $S$ are said to be Morita equivalent if there exists a context $\left[\begin{array}{ll}R & V \\ W & S\end{array}\right]$ in which $V W=R$ and $W V=S$, and the aim of this section is to show that being a PS-ring is a Morita invariant, that is if $R$ and $S$ are Morita equivalent and $R$ is a PS-ring then $S$ is also a PS-ring. In fact we establish the following more general result.

4.1 THEOREM. Suppose $\left[\begin{array}{cc}R & V \\ W & S\end{array}\right]$ is a Morita context such that

(a) $V s W=0, s \in S$, implies $s=0$.

(b) $S / W V$ is flat as a right $S$-module.

(c) $V W r=0, r \in R$, implies $r=0$.

If $R$ is a PS-ring then $S$ is also a PS-ring. In particular, being a PS-ring is a Morita invariant. 
The machinery we use to prove this result has been developed in [10]. Suppose a Morita context $\left[\begin{array}{cc}R & V \\ W & S\end{array}\right]$ is given. If $M$ is a left $R$-module then

$$
M^{\circ}=\left(W \otimes_{R} M\right) / A_{M}(V)
$$

is a left $S$-module, where

$$
A_{M}(V)=\left\{\sum_{i} w_{i} \otimes m_{i} \in W \otimes_{R} M \mid \sum_{i}\left(v w_{i}\right) m_{i}=0 \text { for all } v \in V\right\} .
$$

This construction provides a functor ( $)^{\circ}$ from $R$-mod to $S$-mod which preserves monomorphisms and epimorphisms. The coset $w \otimes m+A_{M}(V)$ in $M^{\circ}$ is denoted by $w \circ m$.

There is a dually defined functor ( $)^{*}: S$-mod $\rightarrow R$-mod and the pair of functors produce a relationship

$$
\theta_{M}:\left(M^{\circ}\right)^{*} \rightarrow M
$$

for each module ${ }_{R} M$ given by $\left(\sum v_{j} * x_{j}\right) \theta_{M}=\sum v_{j} \circ x_{j}$, where $v_{j} \in V$ and $x_{j} \in M^{\circ}$, and where, if $x=\sum w_{i} \circ m_{i}$ then $v \circ x=\sum\left(v w_{i}\right) m_{i}$. Note that $v \circ x=0$ for all $v \in V$ if and only if $x=0$. It is shown in [10] that this is a well-defined map if and only if $V W m \neq 0$ for all $0 \neq m \in(V W) M$, and in this case $\theta_{M}$ is an $R$-monomorphism and $\operatorname{im} \theta_{M}=(V W) M$. Furthermore $\theta_{M}$ is a well-defined map for all $R$-modules $M$ if and only if $R / V W$ is flat as a right $R$-module and, when this holds, $\theta:()^{\circ *} \rightarrow 1_{R \text {-mod }}$ is a natural transformation of functors. The dual relationship is denoted by $\varphi_{N}:\left(N^{*}\right)^{\circ} \rightarrow N$ for all $S$-modules ${ }_{S} N$.

A number of properties are known to pass from $M$ to $M^{\circ}$, sometimes with conditions imposed on the context. However it is always the case that if ${ }_{R} M$ is simple and $M^{\circ} \neq 0$ then $M^{\circ}$ is simple. If ${ }_{R} M$ is faithful and the context satisfies condition (a) in Theorem 4.1 then $M^{\circ}$ is faithful. The first step in our proof of Theorem 4.1 is to show that $M^{\circ}$ is projective under suitable conditions on $M$ and the context.

4.2 LEMMA. Let $\left[\begin{array}{cc}R & V \\ W & S\end{array}\right]$ be a Morita context and let $M$ be a left $R$-module such that $(V W) M=M$ and $\varphi_{M}$ 。 is well-defined (which holds in particular if $S / W V$ is flat as a right $S$-module). Then $\varphi_{M}$ o is an isomorphism.

PROOF. From the results in [10] cited above all that remains is to show that $\operatorname{im} \varphi_{M^{\circ}}=M^{\circ}$, that is that $(W V) M^{\circ}=M^{\circ}$. However $(W V) M^{\circ}=[(W V) M]^{\circ}=$ $M^{\circ}$, so the lemma is proved.

4.3 Proposition. Let $\left[\begin{array}{ll}R & V \\ V & S\end{array}\right]$ be a Morita context such that $S / W V$ is flat as a right $S$-module. Let $M$ be a left $R$-module.

(1) If $M$ is projective and $(V W) M=M$, then ${ }_{S} M^{\circ}$ is projective.

(2) If $\left(M^{\circ}\right)^{*}$ is projective and $\varphi_{M^{\circ}}$ is an isomorphism, then ${ }_{S} M^{\circ}$ is projective.

Proof. (1) By Lemma 4.2, $\varphi_{M^{\circ}}$ has an inverse $\psi: M \rightarrow\left(M^{\circ}\right)^{*}$. Given a diagram

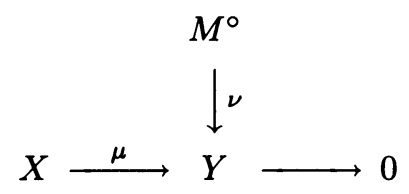


in $S$-mod there exists an $R$-homomorphism $\lambda: M \rightarrow X^{*}$ such that the diagram

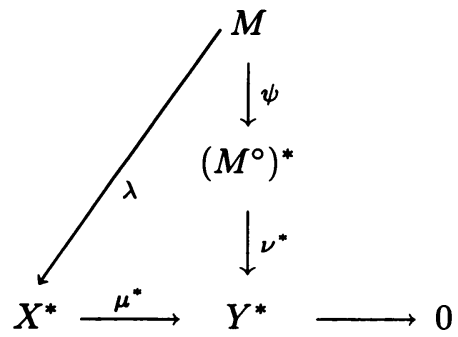

in $R$-mod is commutative. Since $\varphi$ is a natural transformation the diagram

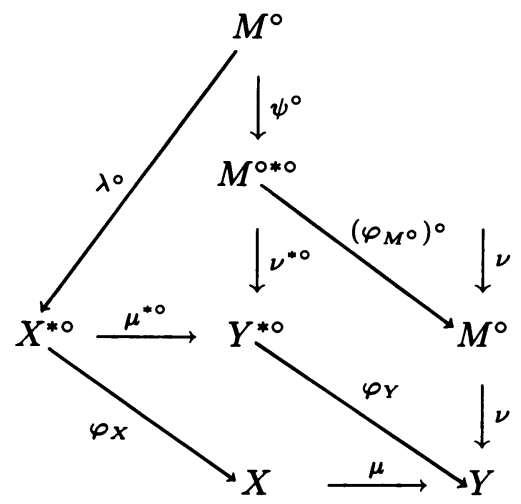

is commutative in $S$-mod. Thus $\lambda^{\circ} \varphi_{X} \mu=\psi^{\circ}\left(\varphi_{M^{\circ}}\right)^{\circ} \nu=\nu$ and it follows that $M^{\circ}$ is projective.

(2) A similar proof works only now we take $\lambda:\left(M^{\circ}\right)^{*} \rightarrow X^{*}$.

4.4 Proposition. Suppose $\left[\begin{array}{cc}R & V \\ W & S\end{array}\right]$ is a Morita context such that

(a) $V s W=0, s \in S$, implies $s=0$; and

(b) $S / W V$ is flat as a right $S$-module.

Let ${ }_{R} M$ be a faithful PS-module such that $\theta_{M}$ is well-defined. Then ${ }_{S} M^{\circ}$ is a faithful PS-module.

ProOF. Condition (a) is enough to ensure that $M^{\circ}$ is faithful (if ${ }_{S} M^{\circ}=0$, $s \in S$, then $(V \circ W) M=0)$. We claim first that $(W V) x=0$ where $x \in M^{\circ}$ implies $x=0$. Indeed,

$$
0=V \circ[(W V) x]=(V W)(V \circ x)
$$

and, since $V \circ x \subseteq(V W) M$, the fact that $\theta_{M}$ is well-defined implies $V \circ x=0$. But then $x=0$ as required.

Now let $s_{S} \subseteq \subseteq M^{\circ}$ be simple. Then $(W V) X \neq 0$ by the above so $(W V) X=X$. In particular $X^{*} \neq 0$ and so $X^{*}$ is simple. But $X^{*}$ is a submodule of $\left(M^{\circ}\right)^{*}$, which is isomorphic to $(V W) M$ under $\theta_{M}$, and so $X^{*}$ is projective because $M$ is a PS-module. Furthermore

$$
(V W) X^{*}=(V W)[V * X]=V *[(W V) X]=V * X=X^{*}
$$

and so $\left(X^{*}\right)^{\circ}$ is projective by Proposition 4.3. But $\varphi_{X}: X^{* \circ} \rightarrow X$ is an isomorphism (using condition (b)) and so $X$ is projective. This shows $M^{\circ}$ is a PS-module and so completes the proof. 
PROOF OF THEOREM 4.1. If $R$ is a PS-ring then ${ }_{R} R$ is a faithful PS-module. Condition (c) in Theorem 4.1 ensures that $\theta_{R}$ is well-defined and so $R^{\circ}$ is a faithful PS-module in $S$-mod by Proposition 4.4. Hence $S$ is a PS-ring by Theorem 2.4.

\section{REFERENCES}

1. S. A. Amitsur, Rings of quotients and Morita contexts, J. Algebra 17 (1971), 273-298.

2. E. P. Armendariz, A note on extensions of Baer and P.P.-rings, J. Austral. Math. Soc. 18 (1974), 470-473.

3. G. Azumaya, Some properties of TTF-classes, Proc. Conf. on Orders, Group Rings and Related Topics, Springer-Verlag, 1973, 72-83.

4. G. Baccella, On C-semisimple rings. A study of the socle of a ring, Comm. Algebra 8 (1980), 889-909.

5. _ Generalized $V$-rings and Von Neumann regular rings, Rend. Sem. Mat. Univ. Padova 72 (1984), 117-133.

6. W. D. Burgess, Mathematical Reviews, 51 \#3224.

7. S. Endo, A note on P.P.-rings (a supplement to Hattori's paper), Nagoya Math. J. 17 (1960), 167-170.

8. R. Gordon, Rings in which minimal left ideals are projective, Pacific J. Math. 31 (1969), 679 692.

9. J. N. Manocha, On rings with essential socle, Comm. Algebra 4 (1976), 1077-1086.

10. W. K. Nicholson and J. F. Watters, Morita context functors, Math. Proc. Cambridge Philos. Soc. (to appear).

11. R. C. Shock, Polynomial rings of finite dimensional rings, Pacific J. Math. 42 (1972), 251-257.

12. J. M. Zelmanowitz, Regular modules, Trans. Amer. Math. Soc. 163 (1972), 341-355.

Department of Mathematics and Statistics, University of Calgary, Calgary, Alberta, CANADA T2N 1N4

Department of MAThematics, University of Leicester, Leicester, England LE1 7RH 